\title{
Organ-Specific Screening for Protein Damage Using Magnetic Bead Bioreactors and LC-MS/MS
}

Di Jiang ${ }^{\dagger}$, Min Shen $^{\dagger}$, Ben Ahiadu ${ }^{\dagger}$ and James F. Rusling ${ }^{*} \neq \S_{\|}$

${ }^{\dagger}$ Department of Chemistry, University of Connecticut, Storrs, Connecticut 06269, United States

${ }^{\ddagger}$ Department of Surgery and Neag Cancer Center, UConn Health, Farmington, Connecticut 06032, United States

$\S$ Institute of Material Science, University of Connecticut, Storrs, Connecticut 06269, United States

" School of Chemistry, National University of Ireland at Galway, Galway H91 TK33, Ireland

* Corresponding Author, email: james.rusling@uconn.edu

Chemicals and Materials. S3

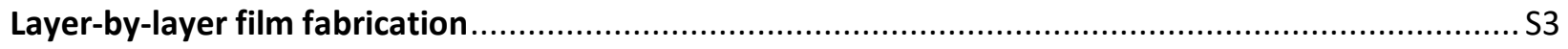

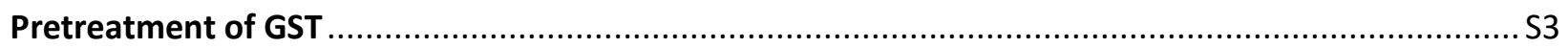

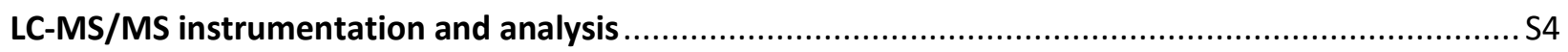

Scheme S1. Collision induced dissociation of peptides resulting in $\mathrm{b}$ and $\mathrm{y}$ ions. .................................S5

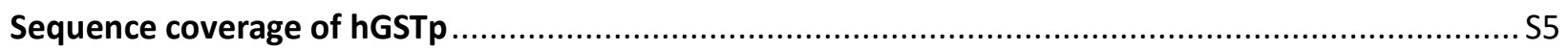

Table S1. UHPLC gradient used for metabolite and tryptic peptide identification and quantitation........ S5

Table S2. MRM transitions and parameters used in the metabolite and peptide quantification.............S6

Table S3. M/z values for NAPQI reacted peptides and unreacted peptides .......................................S7

Figure S1. The representative MRM chromatograms containing two transitions of each molecule for quantitation purpose. (A) NAPQI-GSH conjugate; (B) internal standard $\mathrm{N}^{2}$-benzolyguanosine............... S8

Figure S2. Micro BCA protein assays for (A) standard human liver microsome (HLM), (B) standard

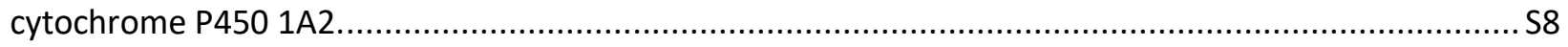

Figure S3. Product ion spectrum of (A) unadducted peptide CAALR with $\mathrm{m} / \mathrm{z} 295.7$; (B) unadducted peptide

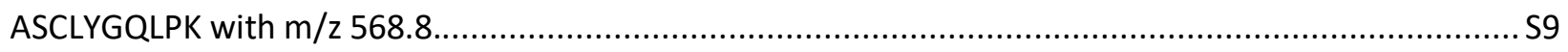

Figure S4. The representative MRM chromatograms containing two transitions for a single tryptic peptide for quantitation purpose. S9

Figure S5. Product ion spectrum of (A) unadducted peptide RCKY with $\mathrm{m} / \mathrm{z} 313.7$; (B) unadducted peptide VLAPGCLD with $\mathrm{m} / \mathrm{z} 422.8$. S10

Figure S6. The representative MRM chromatograms containing two transitions for a single Chymotrypsin digested peptide for quantitation purpose. S10

Figure S7. The representative MRM chromatograms containing two transitions for a single Glu-C digested

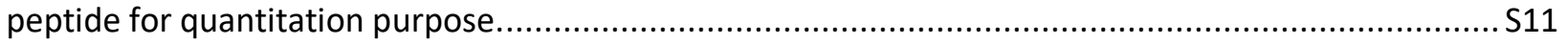

Table S4. MRM transitions and parameters used for NAPQI modified peptides in HSA and BSA...........S12 
Figure S8. Product ion spectra of NAPQI reacted peptides (A) CASLQK with $\mathrm{m} / \mathrm{z} 399.8$; (B) YICENQDSISSK with $\mathrm{m} / \mathrm{z}$ 768.3; (C) DVCK with $\mathrm{m} / \mathrm{z} 307.3$; (D) CCTESLVNR with $\mathrm{m} / \mathrm{z} 611.8$. S13

Figure S9. (A) XIC for NAPQI reacted peptides from BSA. Product ion spectra of NAPQI reacted peptides (B) CASIQK with $\mathrm{m} / \mathrm{z}$ 400.0; (C) GACLLPK with $\mathrm{m} / \mathrm{z}$ 425.4. The amounts of NAPQI reacted DVCK, and YICDNQDTISSK are too small to detect the product ion spectra....................................................... 13

S13

Table S5. MRM transitions and parameters used for raloxifene modified peptides in hGSTp S14

Figure S10. (A) XIC for raloxifene reacted peptides from hGSTp. Product ion spectra of raloxifene reacted peptides (B) CAALR with $\mathrm{m} / \mathrm{z} 335.5, \mathrm{z}=+3$; (C) ASCLYGQLPK with $\mathrm{m} / \mathrm{z} 517.6, \mathrm{z}=+3$. 


\section{Chemicals and Materials}

Reduced glutathione (GSH), acetaminophen, raloxifene hydrochloride, glucose 6-phosphate (G6P), glucose 6-phosphate dehydrogenase (G6PDH), nicotinamide adenine dinucleotide phosphate (NADP ${ }^{+}$, urea, dithiothreitol (DTT), iodoacetamide (IAA), poly(diallyldimethylammonium chloride) (PDDA), poly(sodium 4-styrenesulfonate) (PSS), myoglobin from horse heart were from Millipore Sigma. Human liver microsome (HLM), human lung microsome (HLUM), human kidney microsome (HKM), and human intestine microsome (HIM) were purchased from Sekisui XenoTech, LLC. Human supersomes 2A6, 3A5, 3A4, $2 \mathrm{E} 1$ (Cytochrome P450 + Cytochrome c Reductase + Cytochrome $b_{5}$ ) and human supersomes 1A2, $1 \mathrm{B1}$ (Cytochrome P450 + Cytochrome c Reductase) were from CORNING. Greiner 96 well plates, polypropylene was from Millipore Sigma. AcroPrep advance 96 well filter plates for ultrafiltration, Omega $^{\mathrm{TM}}$ membrane $3 \mathrm{~K}$ MWCO was from Pall corporation.

\section{Layer-by-layer film fabrication}

$40 \mu \mathrm{L}$ of magnetic particles was dispersed in $160 \mu \mathrm{L}$ of $10 \mathrm{mM}$ Tris buffer (pH 7.4, $10 \mathrm{mM} \mathrm{NaCl}$ ). $200 \mu \mathrm{L}$ PDDA ( $2 \mathrm{mg} / \mathrm{mL}, 50 \mathrm{mM} \mathrm{NaCl}$ ) was added drop wisely followed by a 20 min assembly to coat the negatively charged surface with positively charged polycations. The magnetic particles are pulled towards the magnet. Then, the supernatant was discarded, particles were washed with Tris buffer twice to remove loosely bound polycations, and dispersed into $200 \mu \mathrm{L}$ Tris buffer. The same assembly and wash steps were repeated for polyanion PSS ( $3 \mathrm{mg} / \mathrm{mL}, 50 \mathrm{mM} \mathrm{NaCl}$ ) and human microsomes or supersomes film growth. The final architecture on the magnetic bioreactors was MP/PDDA/PSS/PDDA/Microsome or Supersome.

\section{Pretreatment of GST}

$100 \mu \mathrm{g}$ of GST was incubated with $20 \mathrm{mM}$ DTT at 37 for $1 \mathrm{~h}$, then washed twice and concentrated by $3 \mathrm{KDa}$ Ultra centrifugal filter unit, and resuspended in $100 \mu \mathrm{L}$ Tris buffer. 


\section{LC-MS/MS instrumentation and analysis}

LC-MS/MS analysis was done on a Thermo Scientific Dionex Ultimate 3000 UHPLC system interfaced to a Thermo Scientific TSQ Quantiva triple quadrupole mass spectrometer. The metabolites identification was done in the product ion scan mode with collision energy $20 \mathrm{~V}$ for GSH-acetaminophen (m/z 457.2). Multiple reaction monitoring (MRM) mode was used to quantify the metabolites formation. The mass transitions were listed in Table S2. Key parameters of MS instrument were set as ion source type = H-ESI , spray voltage $=3800 \mathrm{~V}$, sheath gas $=15 \mathrm{Arb}$, aux gas $=7 \mathrm{Arb}$, sweep gas $=4 \mathrm{Arb}$, ion transfer tube temperature $=300{ }^{\circ} \mathrm{C}$, vaporizer temperature $=30^{\circ} \mathrm{C}$. MRM properties were set as cycle time $=1 \mathrm{~s}, \mathrm{Q} 1$ resolution $(\mathrm{FWHM})=0.7, \mathrm{Q} 3$ resolution $(\mathrm{FWHM})=0.7, \mathrm{CID}$ gas $=1.5$ mTorr .

The adducted peptide identification was done in the product ion scan mode with collision energy $14 \mathrm{~V}$ for adducted peptide 14-18 (m/z 341.7), $23 \vee$ for adducted peptide 45-54 (m/z 614.8), $20 \vee$ for adducted peptide 100-103 (m/z 359.7), $21 \mathrm{~V}$ for adducted peptide 164-171 (m/z 468.7). Multiple reaction monitoring (MRM) mode was used to quantify the adducted peptides. The mass transitions were listed in Table S2. Key parameters of MS instrument were set as ion source type $=\mathrm{H}-\mathrm{ESI}$, spray voltage $=3900 \mathrm{~V}$, sheath gas $=20 \mathrm{Arb}$, aux gas $=7 \mathrm{Arb}$, sweep gas $=2 \mathrm{Arb}$, ion transfer tube temperature $=300{ }^{\circ} \mathrm{C}$, vaporizer temperature $=30{ }^{\circ} \mathrm{C}$. MRM properties were set as cycle time $=1 \mathrm{~s}, \mathrm{Q} 1$ resolution $(\mathrm{FWHM})=0.7, \mathrm{Q} 3$ resolution $(\mathrm{FWHM})=0.7, \mathrm{CID}$ gas $=1.5$ mTorr .

Collision-induced dissociation (CID) provided information on the sequence of peptides by fragmentation of peptides forming $b$ and $y$ ions (Scheme S1). The position of modifications was determined by detecting the difference in $\mathrm{m} / \mathrm{z}$ values of the $\mathrm{b}$ and $\mathrm{y}$ ions of unreacted and reacted peptides. For example, if amino acid $R_{3}$ was modified with NAPQI, the $m / z$ values of $b_{3}, b_{4}, y_{3}$, and $y_{4}$ will increase. 


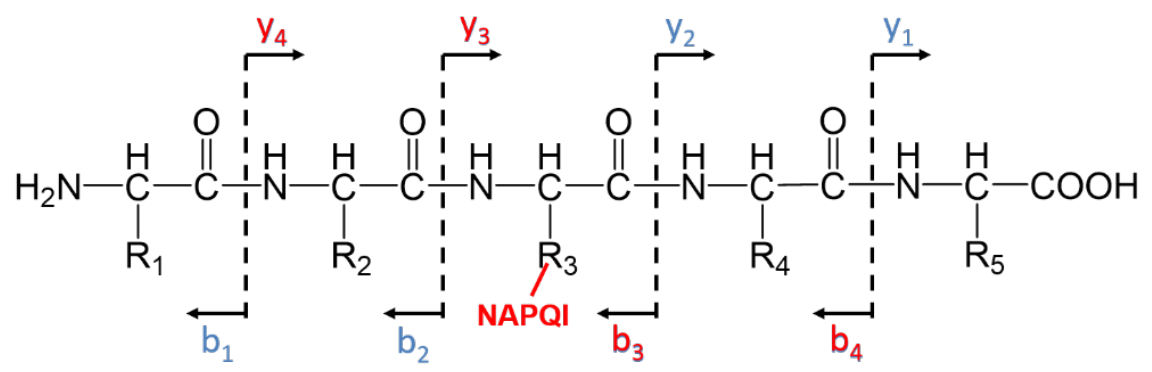

Scheme S1. Collision induced dissociation of peptides resulting in b and y ions.

\section{Sequence coverage of hGSTp}

Sequence coverage was estimated by monitoring digested peptides with triple quadrupole mass spectrometry. For trypsin digestion, the sequence coverage was calculated as $78 \%$. Amino acids 12,13 , $101,102,141-182$ were not covered. Cys-101, Cys-169 were not covered by trypsin digestion. For Glu-C digestion, the sequence coverage was calculated as 58\%. Peptide containing Cys-169 (VLAPGCLD) was covered. For chymotrypsin, the sequence coverage was calculated as $63 \%$. Peptide containing Cys-101 (RCKY) was covered.

Table S1. UHPLC gradient used for metabolite and tryptic peptide identification and quantitation.

\begin{tabular}{|c|c|c|c|c|c|}
\hline \multicolumn{4}{|c|}{ Metabolite separation gradient } & \multicolumn{3}{c|}{ Peptide separation gradient } \\
\hline Time $(\mathrm{min})$ & Flow $(\mu \mathrm{L} / \mathrm{min})$ & $\% \mathrm{~B}$ & Time $(\mathrm{min})$ & Flow $(\mu \mathrm{L} / \mathrm{min})$ & $\% \mathrm{~B}$ \\
\hline 0 & 6 & 1 & 0 & 15 & 1 \\
\hline 7 & 6 & 1 & 7 & 15 & 1 \\
\hline 16 & 6 & 50 & 19 & 15 & 30 \\
\hline 16.5 & 6 & 90 & 19.5 & 15 & 90 \\
\hline 21 & 6 & 90 & 21 & 15 & 90 \\
\hline 21.5 & 6 & 1 & 21.5 & 15 & 1 \\
\hline 25 & 6 & 1 & 25 & 15 & 1 \\
\hline
\end{tabular}


Table S2. MRM transitions and parameters used in the metabolite and peptide quantification.

\begin{tabular}{|c|c|c|c|}
\hline Metabolites & $\begin{array}{l}\text { Collision energy } \\
(\mathrm{CE}), \mathrm{V}\end{array}$ & Mass transition, $\mathrm{m} / \mathrm{z}$ & Role \\
\hline \multirow[t]{2}{*}{ NAPQI-GSH } & 18 & $457.2 \rightarrow 328.3$ & Quantitation \\
\hline & 20 & $457.2 \rightarrow 382.2$ & Confirmation \\
\hline \multirow[t]{2}{*}{$\mathrm{N}^{2}$-benzolyguanosine } & 28 & $388.1 \rightarrow 238.1$ & Quantitation \\
\hline & 35 & $388.1 \longrightarrow 256.1$ & Confirmation \\
\hline Peptides & $\mathrm{CE}, \mathrm{V}$ & Mass transition, $\mathrm{m} / \mathrm{z}$ & Role \\
\hline \multirow[t]{2}{*}{ CAALR } & 12.4 & $295.7(+2) \rightarrow 359.2(+1)(y 3)$ & Quantitation \\
\hline & 12.4 & $295.7(+2) \rightarrow 430.3(+1)(y 4)$ & Confirmation \\
\hline \multirow[t]{2}{*}{ CAALR } & 13.9 & $341.7(+2) \rightarrow 359.2(+1)(y 3)$ & Quantitation \\
\hline & 13.9 & $341.7(+2) \rightarrow 430.3(+1)(y 4)$ & Confirmation \\
\hline \multirow[t]{2}{*}{ ASCLYGQLPK } & 21.6 & $568.8(+2) \rightarrow 705.4(+1)(y 6)$ & Quantitation \\
\hline & 21.6 & $568.8(+2) \rightarrow 542.3(+1)(y 5)$ & Confirmation \\
\hline \multirow[t]{2}{*}{ ASCLYGQLPK } & 23.2 & $614.8(+2) \rightarrow 705.4(+1)(y 6)$ & Quantitation \\
\hline & 23.2 & $614.8(+2) \rightarrow 542.3(+1)(y 5)$ & Confirmation \\
\hline \multirow[t]{2}{*}{ LFTGHPETLEK } & 23.9 & $636.3(+2) \rightarrow 716.4(+1)(y 6)$ & Quantitation \\
\hline & 23.9 & $636.3(+2) \rightarrow 910.5(+1)(y 8)$ & Confirmation \\
\hline \multirow[t]{2}{*}{ RCKY } & 13 & $313.7(+2) \rightarrow 182.1(+1)(y 1)$ & Quantitation \\
\hline & 13 & $313.7(+2) \rightarrow 445.2(+1)(b 3)$ & Confirmation \\
\hline \multirow[t]{2}{*}{ RCKY } & 14.6 & $359.7(+2) \rightarrow 182.1(+1)(y 1)$ & Quantitation \\
\hline & 14.6 & $359.7(+2) \rightarrow 537.3(+1)(b 3)$ & Confirmation \\
\hline
\end{tabular}




\begin{tabular}{|l|l|l|l|}
\hline KKHGTVVL & 17.3 & $441.3(+2) \rightarrow 625.4(+1)(\mathrm{y} 6)$ & Quantitation \\
\hline & 17.3 & $441.3(+2) \rightarrow 753.5(+1)(\mathrm{y} 7)$ & Confirmation \\
\hline VLAPGCLD & 16.9 & $422.7(+2) \rightarrow 213.2(+1)(\mathrm{b} 2)$ & Quantitation \\
\hline & 16.9 & $422.7(+2) \rightarrow 561.2(+1)(\mathrm{y} 5)$ & Confirmation \\
\hline VLAPGCLD & 18.2 & $468.7(+2) \rightarrow 213.2(+1)(\mathrm{b} 2)$ & Quantitation \\
\hline & 18.2 & $468.7(+2) \rightarrow 653.3(+1)(\mathrm{y} 5)$ & Confirmation \\
\hline IAAKYKE & 16.3 & $411.7(+2) \rightarrow 638.4(+1)(\mathrm{y} 5)$ & Quantitation \\
\hline & 16.3 & $411.7(+2) \rightarrow 439.2(+1)(\mathrm{y} 3)$ & Confirmation \\
\hline
\end{tabular}

Table S3. M/z values for NAPQI reacted peptides and unreacted peptides

\begin{tabular}{|l|l|l|}
\hline & NAPQI reacted peptides, $\mathrm{m} / \mathrm{z}, \mathrm{z}=+2$ & Unreacted peptides with IAA, $\mathrm{m} / \mathrm{z}, \mathrm{z}=+2$ \\
\hline CAALR & 341.7 & 295.7 \\
\hline ASCLYGQLPK & 614.8 & 568.8 \\
\hline RCKY & 359.7 & 313.7 \\
\hline VLAPGCLD & 468.7 & 422.7 \\
\hline
\end{tabular}



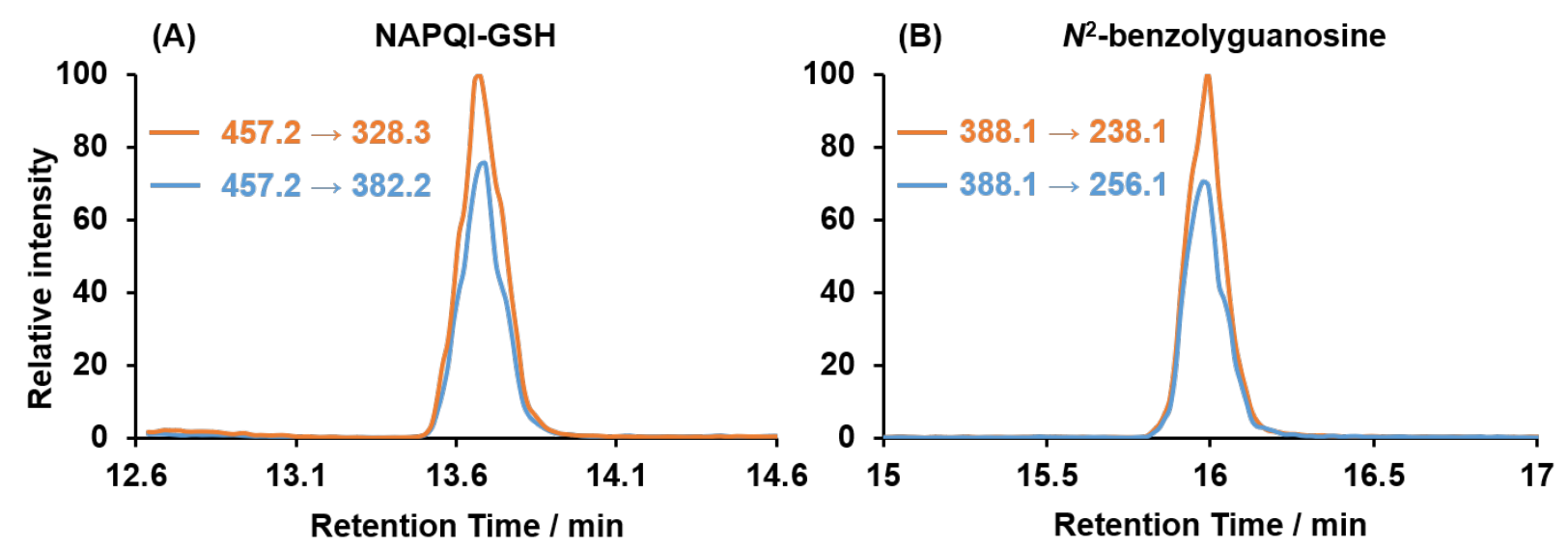

Figure S1. The representative MRM chromatograms containing two transitions of each molecule for quantitation purpose. (A) NAPQI-GSH conjugate; (B) internal standard $\mathrm{N}^{2}$-benzoylguanosine
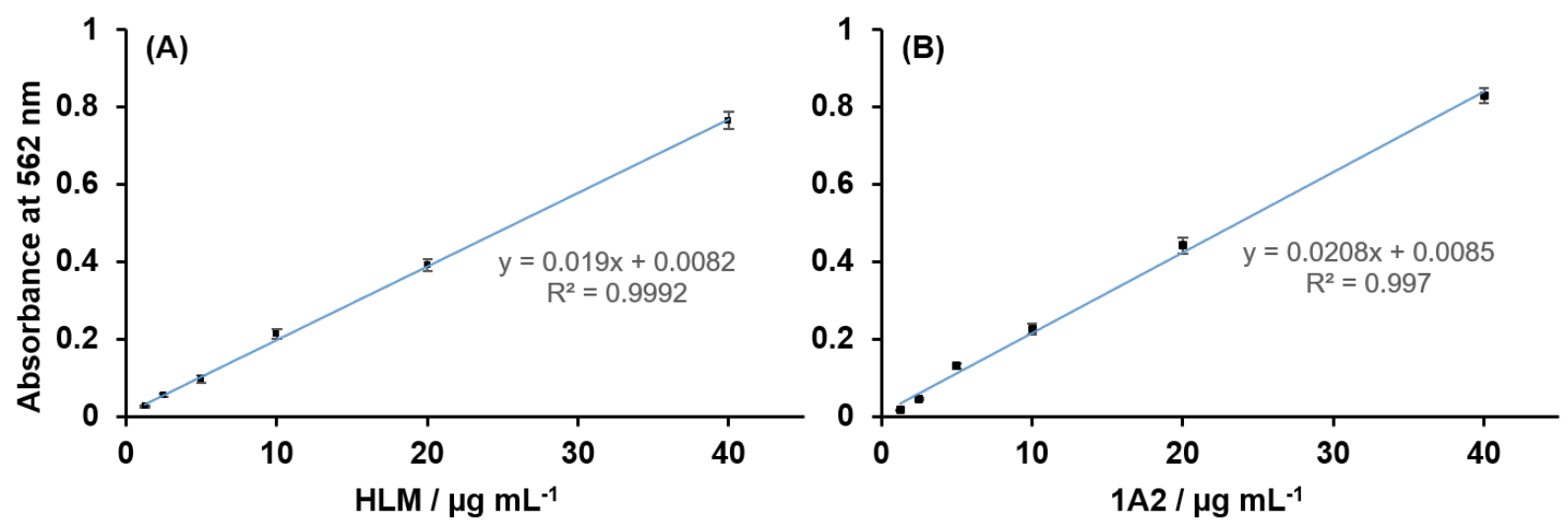

Figure S2. Micro BCA protein assays for (A) standard human liver microsome (HLM), (B) standard cytochrome P450 $1 \mathrm{~A} 2$. 

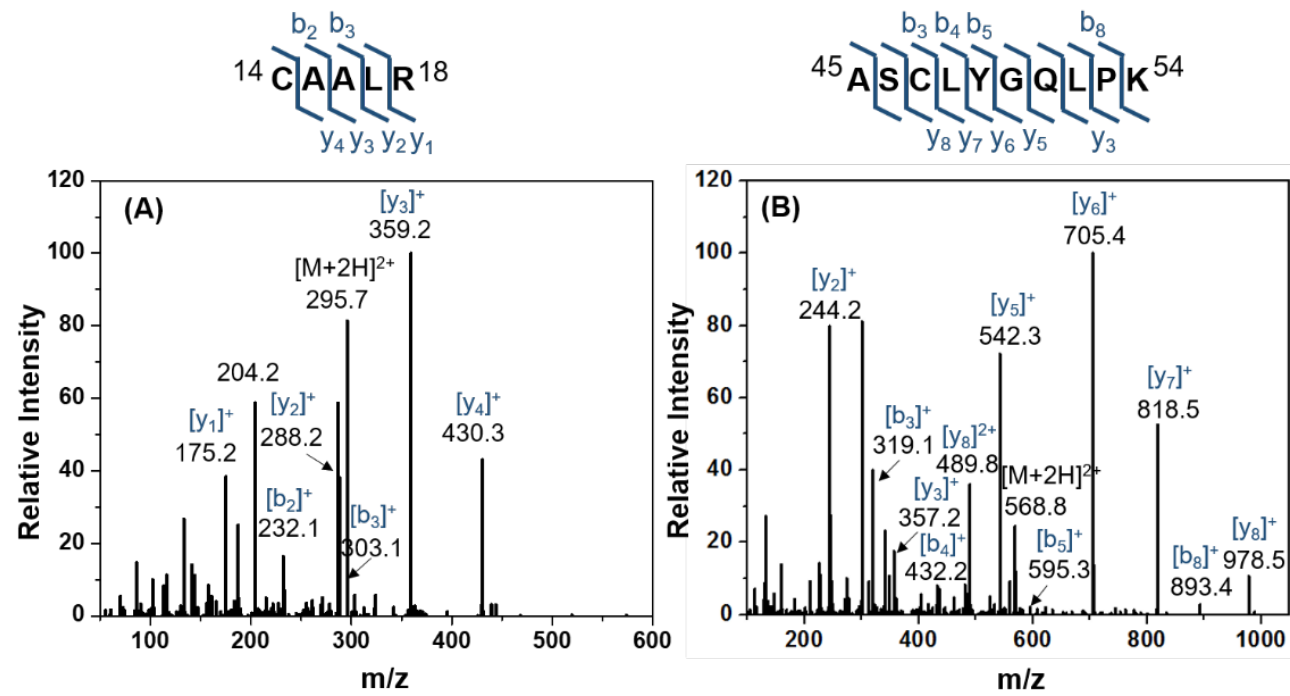

Figure S3. Product ion spectrum of (A) unadducted peptide CAALR with $\mathrm{m} / \mathrm{z} 295.7$; (B) unadducted peptide ASCLYGQLPK with $\mathrm{m} / \mathrm{z} 568.8$.

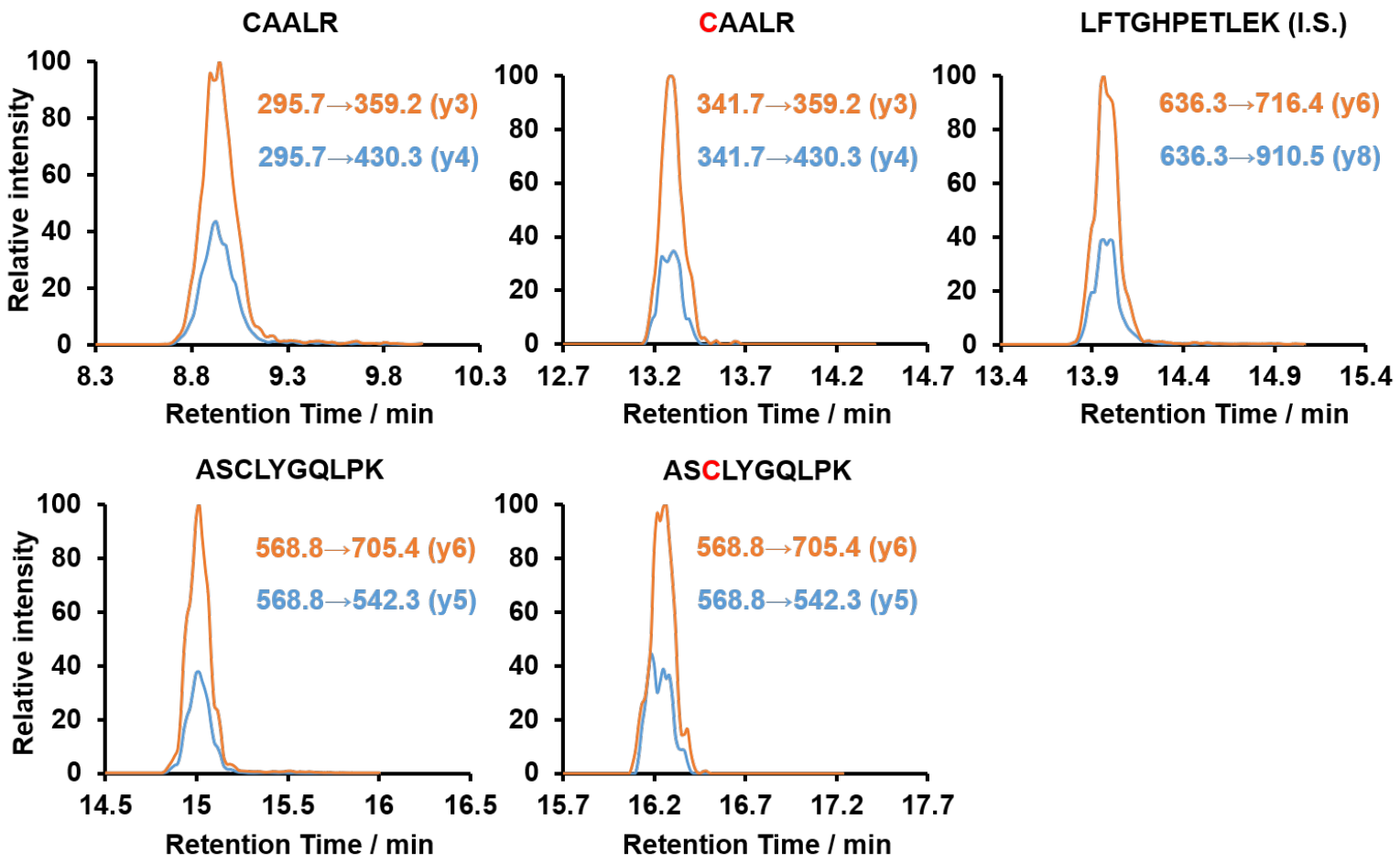

Figure S4. The representative MRM chromatograms containing two transitions for a single tryptic peptide for quantitation purpose. 

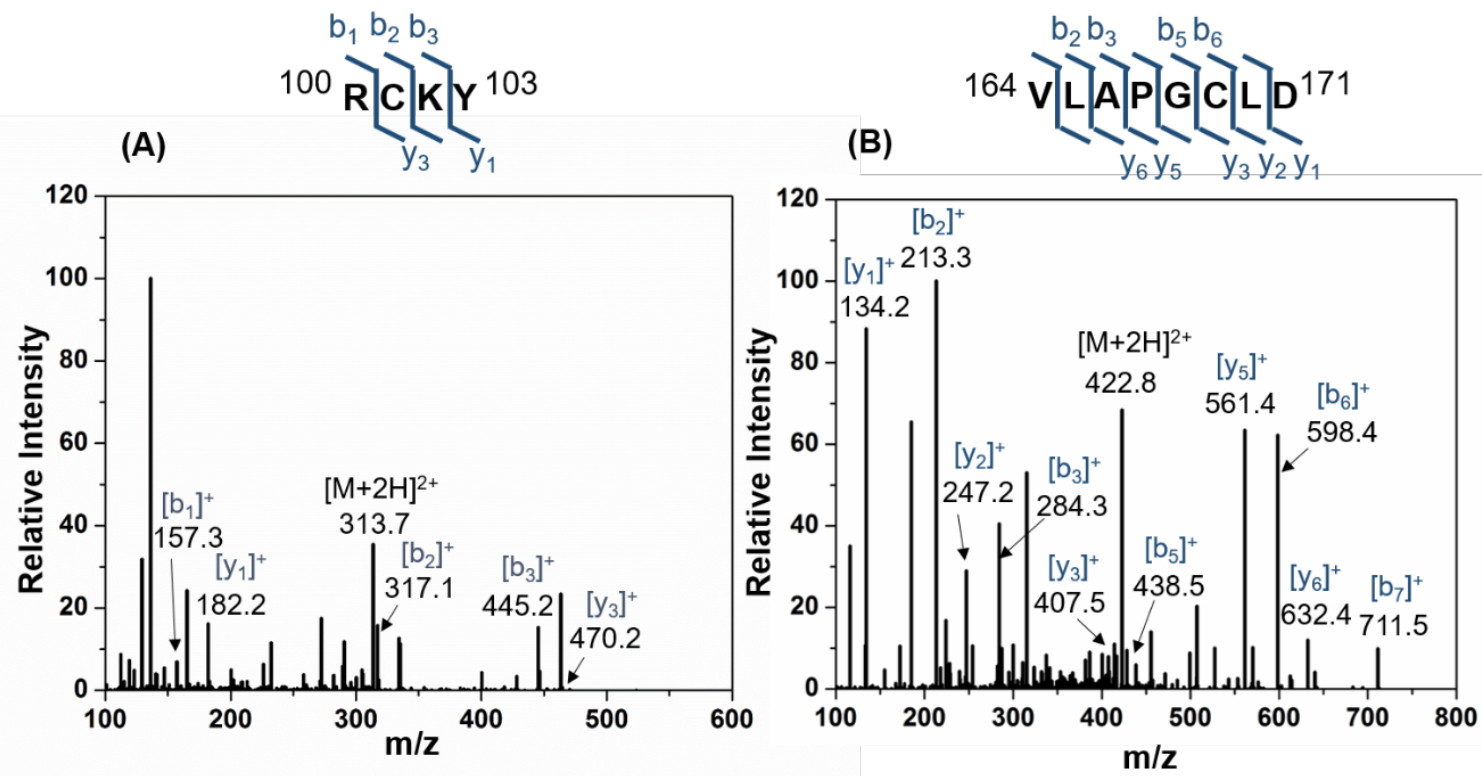

Figure S5. Product ion spectrum of (A) unadducted peptide RCKY with $\mathrm{m} / \mathrm{z} 313.7$; (B) unadducted peptide VLAPGCLD with $\mathrm{m} / \mathrm{z} 422.8$.

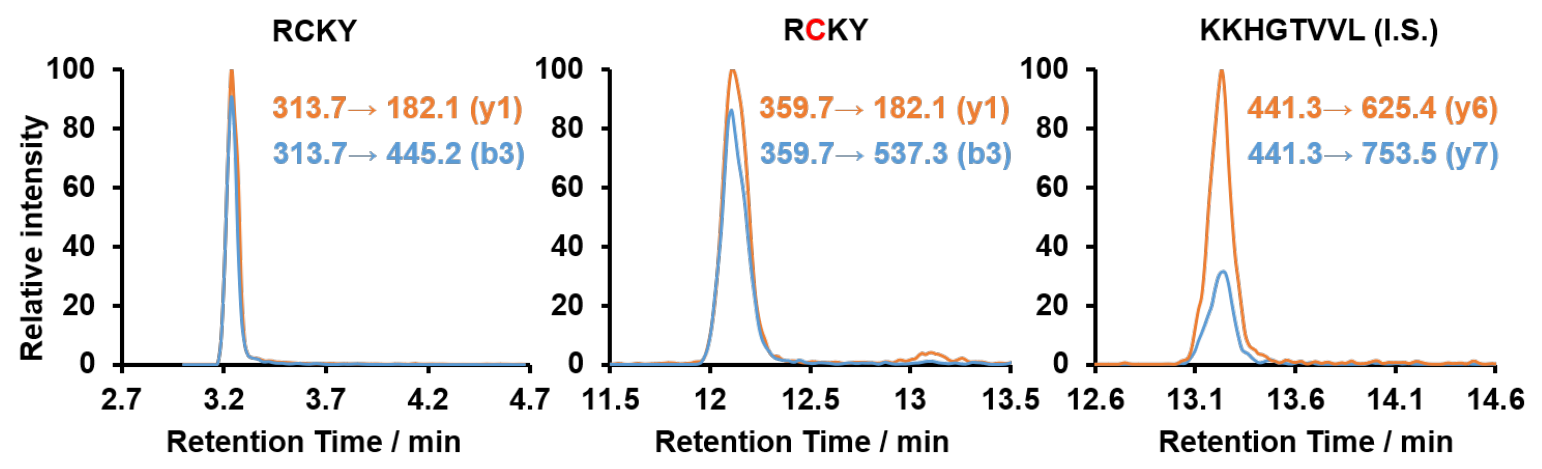

Figure S6. The representative MRM chromatograms containing two transitions for a single Chymotrypsin digested peptide for quantitation purpose. 


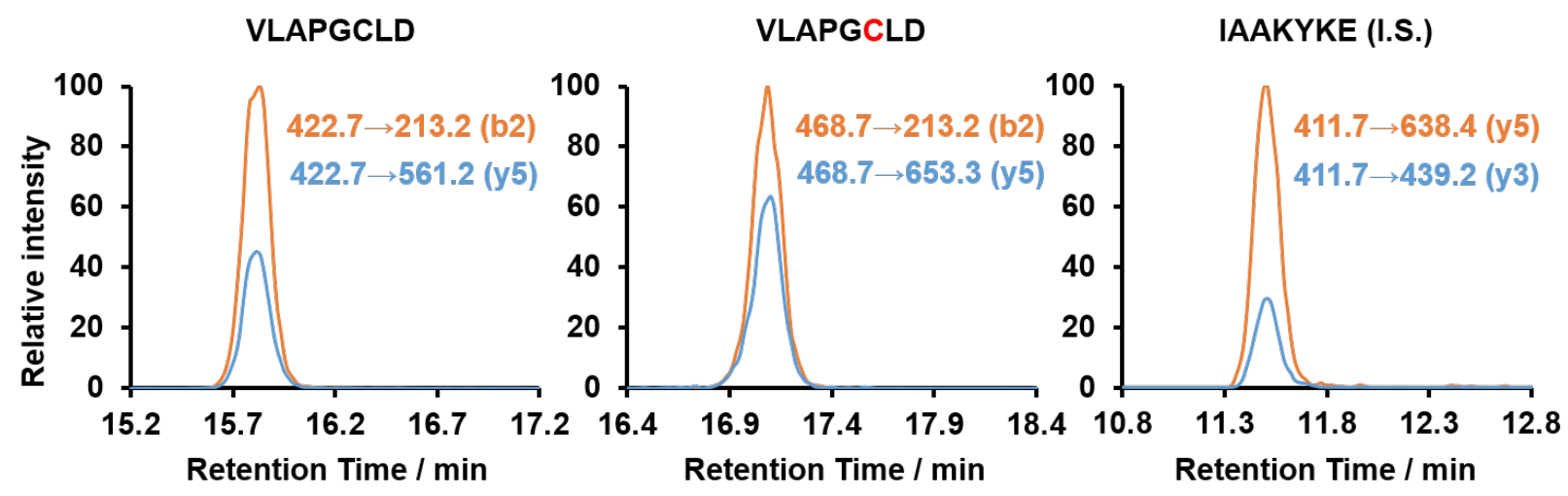

Figure S7. The representative MRM chromatograms containing two transitions for a single Glu-C digested peptide for quantitation purpose. 
Table S4. MRM transitions and parameters used for NAPQI modified peptides in HSA and BSA

\begin{tabular}{|c|c|c|}
\hline \multicolumn{3}{|c|}{ HSA } \\
\hline NAPQI modified Peptides & Collision energy (CE), $\mathrm{V}$ & Mass transitions, $\mathrm{m} / \mathrm{z}$ \\
\hline \multirow[t]{2}{*}{ LCTVATLR } & 19.8 & $513.3(+2) \rightarrow 660.4(+1)(y 6)$ \\
\hline & 19.8 & $513.3(+2) \rightarrow 559.3(+1)(y 5)$ \\
\hline \multirow[t]{2}{*}{ NECFLQHK } & 22.2 & $584.3(+2) \rightarrow 924.4(+1)(y 6)$ \\
\hline & 22.2 & $584.3(+2) \rightarrow 672.3(+1)(y 5)$ \\
\hline \multirow[t]{2}{*}{ AACLLPK } & 17 & $432.6(+2) \rightarrow 793.4(+1)(y 6)$ \\
\hline & 17 & $432.6(+2) \rightarrow 722.4(+1)(y 5)$ \\
\hline \multirow[t]{2}{*}{ CASLQK } & 15.9 & $399.8(+2) \rightarrow 546.3(+1)(y 5)$ \\
\hline & 15.9 & $399.8(+2) \rightarrow 475.5(+1)(y 4)$ \\
\hline \multirow[t]{2}{*}{ YICENQDSISSK } & 28.4 & $768.3(+2) \rightarrow 878.4(+1)(y 8)$ \\
\hline & 28.4 & $768.3(+2) \rightarrow 764.4(+1)(y 7)$ \\
\hline \multirow[t]{2}{*}{ DVCK } & 11.2 & $307.3(+2) \rightarrow 498.3(+1)(y 3)$ \\
\hline & 11.2 & $307.3(+2) \rightarrow 399.2(+1)(y 2)$ \\
\hline \multirow[t]{2}{*}{ CCTESLVNR } & 24.8 & $661.8(+2) \rightarrow 818.3(+1)(y 7)$ \\
\hline & 24.8 & $661.8(+2) \rightarrow 717.4(+1)(y 6)$ \\
\hline \multicolumn{3}{|c|}{ BSA } \\
\hline \multirow[t]{2}{*}{ GACLLPK } & 16.8 & $425.8(+2) \rightarrow 722.2(+1)(y 5)$ \\
\hline & 16.8 & $425.8(+2) \rightarrow 470.4(+1)(y 4)$ \\
\hline \multirow[t]{2}{*}{ CASIQK } & 15.9 & $400.0(+2) \rightarrow 546.3(+1)(y 5)$ \\
\hline & 15.9 & $400.0(+2) \rightarrow 475.2(+1)(y 4)$ \\
\hline
\end{tabular}



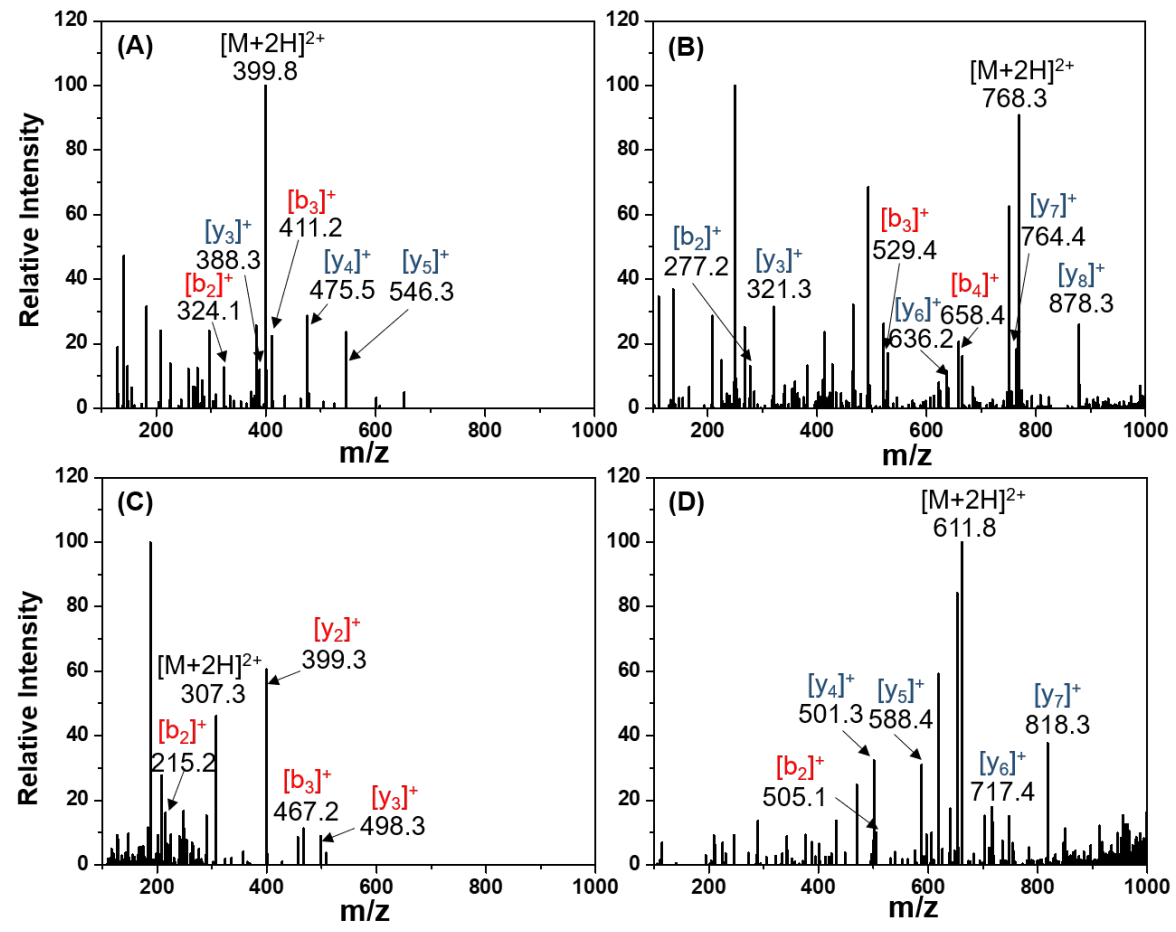

Figure S8. Product ion spectra of NAPQI reacted peptides (A) CASLQK with $\mathrm{m} / \mathrm{z}$ 399.8; (B) YICENQDSISSK with $\mathrm{m} / \mathrm{z}$ 768.3; (C) DVCK with $\mathrm{m} / \mathrm{z}$ 307.3; (D) CCTESLVNR with $\mathrm{m} / \mathrm{z} 611.8$.
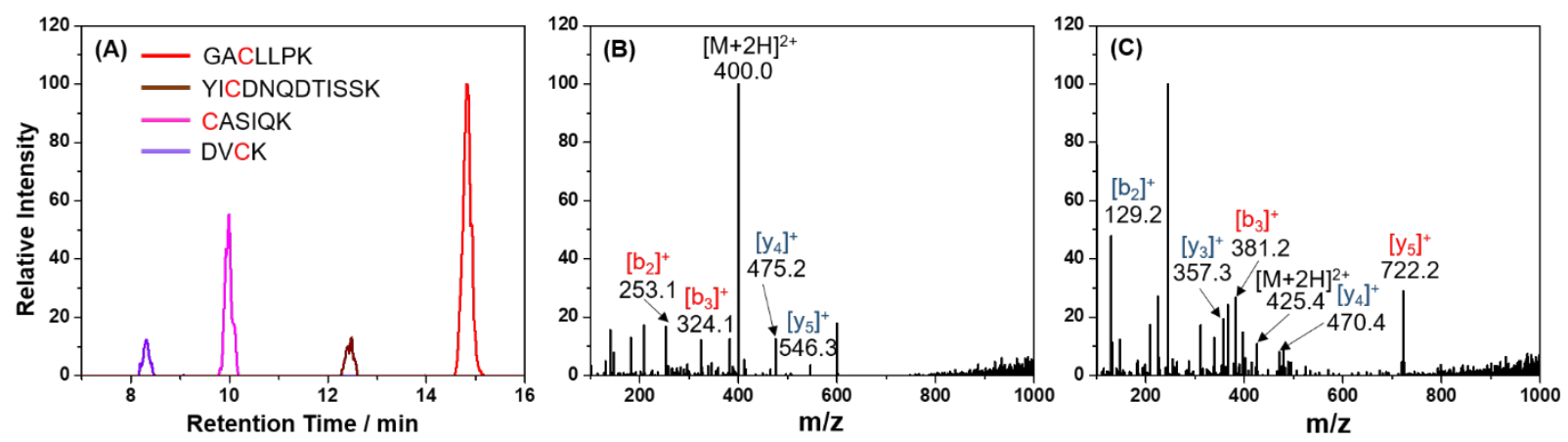

Figure S9. (A) XIC for NAPQI reacted peptides from BSA. Product ion spectra of NAPQI reacted peptides (B) CASIQK with $\mathrm{m} / \mathrm{z}$ 400.0; (C) GACLLPK with $\mathrm{m} / \mathrm{z}$ 425.4. The amounts of NAPQI reacted DVCK, and YICDNQDTISSK are too small to detect the product ion spectra. 
Table S5. MRM transitions and parameters used for raloxifene modified peptides in hGSTp

\begin{tabular}{|l|l|l|}
\hline raloxifene modified Peptides & Collision energy (CE), V & Mass transitions, $\mathrm{m} / \mathrm{z}$ \\
\hline CAALR & 11.4 & $335.5(+3) \rightarrow 359.2(+1)(\mathrm{y} 3)$ \\
\hline & 11.4 & $335.5(+3) \rightarrow 288.2(+1)(\mathrm{y} 2)$ \\
\hline ASCLYGQLPK & 16.8 & $517.6(+3) \rightarrow 705.4(+1)(\mathrm{y} 6)$ \\
\hline & 16.8 & $517.6(+3) \rightarrow 542.3(+1)(\mathrm{y} 5)$ \\
\hline
\end{tabular}

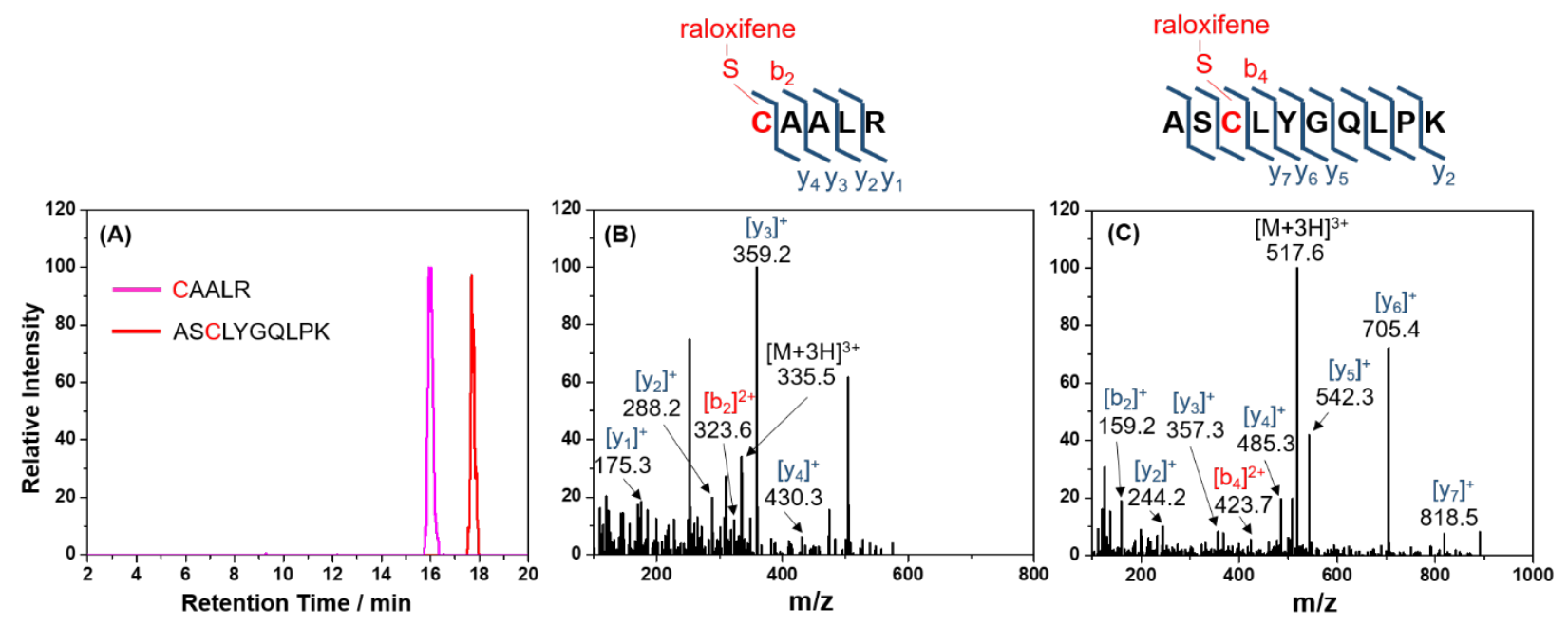

Figure S10. (A) XIC for raloxifene reacted peptides from hGSTp. Product ion spectra of raloxifene reacted peptides (B) CAALR with $\mathrm{m} / \mathrm{z} 335.5, \mathrm{z}=+3$; (C) ASCLYGQLPK with $\mathrm{m} / \mathrm{z} 517.6, \mathrm{z}=+3$. 